\title{
Site-specific bonding of copper adatoms to pyridine end groups mediating the formation of two-dimensional coordination networks on metal surfaces
}

\author{
T. R. Umbach, ${ }^{1}$ M. Bernien, ${ }^{1}$ C. F. Hermanns, ${ }^{1}$ L. L. Sun, ${ }^{2}$ H. Mohrmann, ${ }^{1}$ K. E. Hermann, ${ }^{2}$ A. Krüger, ${ }^{1}$ N. Krane, ${ }^{1}$ \\ Z. Yang (杨泽超), ${ }^{1}$ F. Nickel, ${ }^{1}$ Y.-M. Chang (張銀銘), ${ }^{1}$ K. J. Franke, ${ }^{1}$ J. I. Pascual, ${ }^{3,4}$ and W. Kuch ${ }^{1, *}$ \\ ${ }^{1}$ Institut für Experimentalphysik, Freie Universität Berlin, Arnimallee 14, 14195 Berlin, Germany \\ ${ }^{2}$ Inorg. Chem. Dept., Fritz-Haber-Institut der MPG, Faradayweg 4-6, 14195 Berlin, Germany \\ ${ }^{3}$ CIC nanogune, Tolosa Hiribidea 76, 20018 Donostia-San Sebastian, Spain \\ ${ }^{4}$ Ikerbasque, Basque Foundation for Science, 48011 Bilbao, Spain
}

(Received 26 March 2014; revised manuscript received 14 May 2014; published 9 June 2014)

\begin{abstract}
We study the formation of a coordination network consisting of the organic pyridine-based 2,4,6-tris(4pyridine)-1,3,5-triazine (T4PT) species and $\mathrm{Cu}$ atoms on $\mathrm{Cu}(111)$ and $\mathrm{Ag}(111)$ metal surfaces. Using scanning tunneling microscopy, we find that the organic molecule T4PT forms stable two-dimensional porous networks on the surface of $\mathrm{Cu}(111)$ and, by codeposition of $\mathrm{Cu}$ atoms, also on the $\mathrm{Ag}(111)$ crystal, in which $\mathrm{Cu}$ atoms are twofold coordinated by T4PT molecules. X-ray absorption spectroscopy measurements of the metal-organic network $\mathrm{Cu}-\mathrm{T} 4 \mathrm{PT}$ on $\mathrm{Ag}(111)$ accompanied by density-functional theory calculations show that the nitrogen atoms of the pyridine end groups of the T4PT molecules are the active sites in coordinating the $\mathrm{Cu}$ adatoms. $\mathrm{X}$-ray magnetic circular dichroism experiments reveal that the $\mathrm{Cu}$ atom in such a metal-organic motif is in a low-valent $d^{10}$ state and has no magnetic moment.
\end{abstract}

DOI: 10.1103/PhysRevB.89.235409

PACS number(s): 68.43.-h, 68.37.Ef, 78.70.Dm, 73.20.Hb

\section{INTRODUCTION}

The fabrication of metal-organic networks on metal surfaces with tunable structures and functionalities is one of the key issues for controlling the surface properties of materials on the nanoscale. Self-assembled metal-organic networks consisting of molecular linkers and metal species provide longrange order [1-15] and a programmable network architecture based on the interplay of the functionalized parts of the organic molecule and the metal species. With the choice of the symmetry and size of the organic linker, the geometry of the metal-organic network can be tuned $[5,6]$. Interesting applications of such kinds of networks comprise adjustable templates with adsorption cavities $[7,8]$. Aside from the mere geometry, the electronic properties at the interface to a metallic substrate may be patterned [9]. Intriguing further aspects arise if the transition-metal core exhibits a magnetic moment [10]. The organic linker may then mediate a superexchange coupling leading to metal-organic ferromagnetism [11,12].

To find the best design strategies for metal-organic networks with the desired structural, electronic, and magnetic properties, the metal-organic bonding characteristics, such as coordination symmetry, oxidation state, and magnetic moment, need to be fully understood. As a model system, we choose an organic linker with potentially two active sites for metal coordination. 2,4,6-tris(4-pyridine)-1,3,5-triazine (T4PT) is a triangular molecule and its chemical structure is shown in the inset of Fig. 1(a). The nitrogen sites of the pyridine rings are known to form metal-organic bonds with transition-metal atoms via their lone-pair electrons [12-16]. In T4PT also the lone-pair electrons of the triazine nitrogen atoms may participate in forming bonds. In this study, we investigate the structural, electronic, and magnetic properties of the metal-organic network of T4PT molecules and $\mathrm{Cu}$ atoms.

*kuch@ physik.fu-berlin.de
Using scanning tunneling microscopy (STM), we find that $\mathrm{Cu}$ atoms from $\mathrm{a} \mathrm{Cu}(111)$ surface or codeposited on a $\mathrm{Ag}(111)$ surface together with T4PT molecules self-assemble and form identical ordered metal-organic networks. X-ray absorption spectroscopy (XAS) combined with density-functional theory (DFT) reveal a site-specific coordination of the $\mathrm{Cu}$ atoms solely to the N-lone-pair electrons at the pyridine terminations, whereas the triazine unit remains uncoordinated. The formation of this metal-ligand bond is driving the formation of the two-dimensional metal-organic network at the metal surface. Further, XAS and x-ray magnetic circular dichroism (XMCD) measurements reveal that $\mathrm{Cu}$ atoms inside the metal-organic $\mathrm{Cu}$-T4PT network on $\mathrm{Ag}(111)$ are in a $d^{10}$ state. This state of low covalency of the $\mathrm{Cu}$ ions indicates that only the $4 \mathrm{~s}$ electron is involved in the formation of the metal-organic bond.

\section{EXPERIMENT}

All experiments were carried out in ultrahigh vacuum (UHV) using different setups in order to perform STM or XAS experiments. Atomically clean $\mathrm{Ag}(111)$ and $\mathrm{Cu}(111)$ single-crystal surfaces were obtained by repeated $\mathrm{Ne}^{+}$or $\mathrm{Ar}^{+}$sputtering and annealing cycles under ultrahigh vacuum conditions. T4PT molecules were evaporated from a Knudsen cell at a temperature of $410 \mathrm{~K}$ onto the $\mathrm{Cu}(111)$ and $\mathrm{Ag}(111)$ metal surface while monitoring the deposition rate with a quartz microbalance. In the case of $\operatorname{Ag}(111)$, we evaporated subsequently $\mathrm{Cu}$ atoms by electron bombardment from a molybdenum crucible filled with high-purity $(99.995 \%) \mathrm{Cu}$ material. All preparations were carried out with the sample held at room temperature, unless otherwise stated.

The STM experiments were performed at $5 \mathrm{~K}$ under ultrahigh vacuum conditions. X-ray absorption experiments were carried out using linearly $p$ - and circularly polarized $x$ rays of the beamlines PM3 (Figs. 5 and 6) and UE46-PGM1 (Fig. 10) at the synchrotron radiation source BESSY II in 
Berlin, respectively. Linear and circular degrees of polarization were about $99 \%$ and $85 \%$, respectively. For both beamlines, the photon flux density was in the range of approximately $10^{13}$ photons $\mathrm{s}^{-1} \mathrm{~cm}^{-2}$, while the spot size of the $\mathrm{x}$ rays on the sample was around 0.15 and $0.50 \mathrm{~mm}^{2}$, respectively. Energy resolutions were set to approximately 150, 200, and $300 \mathrm{meV}$ at the $\mathrm{C} K, \mathrm{~N} K$, and $\mathrm{Cu} L_{2,3}$ edges, respectively. Spectra were acquired in total-electron-yield mode and normalized to a reference signal, as well as to the corresponding spectra of the substrate without adsorbed molecules. For the measurements at PM3 and UE46-PGM1, the signal of a gold grid and the last refocusing mirror was used as reference, respectively. Radiation damage could be excluded from comparison of spectra taken immediately after sample preparation and at later times. Isotropic spectra were recorded with linearly $p$-polarized $\mathrm{x}$ rays at the magic angle of incidence, that is, $54.7^{\circ}$ between the $k$ vector of the $\mathrm{x}$ rays and the surface. At this angle, the XA resonance intensities are independent of the orientations of the molecular orbitals.

\section{THEORY}

Quantum chemical calculations using the STOBE code [17] were performed in order to obtain theoretical polarizationresolved XA spectra. The calculations are based on density-functional theory (DFT) in combination with the gradient-corrected revised Perdew-Burke-Ernzerhof (RPBE) exchange-correlation functional $[18,19]$. For the geometry optimization in the electronic ground state, Kohn-Sham orbitals are represented by extended basis sets of contracted Gaussians, where the component atoms, copper, carbon, nitrogen, and hydrogen, are represented by all-electron double-zeta-valenceplus-polarization (DZVP) basis sets.

The computation of theoretical x-ray absorption spectra of the different clusters considers core to unoccupied orbital excitations resulting from dipole transitions [20]. Thus, polarization-resolved spectral intensities $I(E, \vec{e})$ are determined by corresponding dipole transition matrix elements, vectors $\vec{m}=\left(m_{x}, m_{y}, m_{z}\right)$, together with angle-dependent factors of the incoming radiation, characterized by the polarization vector $\vec{e}=\left(e_{x}, e_{y}, e_{z}\right)$ (see Supplemental Material for further details [21]).

The evaluation of all core-excited final states with corresponding transition energies $E$ and matrix elements $m$ is achieved within the transition potential approach [22] in combination with a double-basis-set technique [23]. This approximation can account for partial electronic relaxation due to the presence of the excited electron [24]. The transition energies and corresponding dipole transition matrix elements are convoluted using Gaussian broadening of varying width to simulate instrumental, vibrational, and lifetime broadening. A full-width-at-half-maximum (FWHM) value of $0.7 \mathrm{eV}$ is applied below the ionization threshold, while the broadening is increased linearly by $0.4 \mathrm{eV}$ per eV above threshold.

In the transition potential approach, the electronic core hole relaxation of the excited final state is not fully accounted for. This incomplete relaxation can be corrected in an approximate way by shifting all excitation energies by the difference of the ionization potential computed with the transition potential method and the corresponding value from $\Delta$ Kohn-Sham $(\triangle \mathrm{SCF})$ calculations. This results in a global downward shift of about 1.5 to $1.8 \mathrm{eV}$ for nitrogen and 1.3 to $1.7 \mathrm{eV}$ for carbon. Further, relativistic corrections are included by applying an additional upward shift of the computed spectra by $0.18 \mathrm{eV}$ for nitrogen and $0.08 \mathrm{eV}$ for carbon [25]. For further details of the method, consult Refs. [22,23,26-28].

In the evaluation of x-ray absorption spectra referring to carbon and nitrogen core excitations different basis sets apart from the DZVP sets used for the geometry optimizations are applied for those atoms where the excitations initiate from. At the core-excited carbon or nitrogen centers IGLO-III type ([7s6p2d]) basis sets [29] are used in order to adequately describe inner-shell relaxation effects while the other carbon/nitrogen centers in the clusters are accounted for by effective core potentials (ECPs) for the $1 s$ shell and $[3 s 3 p 1 d]$ valence basis sets to avoid $1 s$ core-orbital mixing [30,31] (see also Ref. [32]).

\section{RESULTS AND DISCUSSION}

\section{A. Structure of the $\mathrm{Cu}$-T4PT coordination network}

We first investigate the structural and electronic properties of the metal-organic networks using scanning tunneling microscopy and spectroscopy. This allows us to resolve the structure and the bonding scheme of the metal-organic coordination network. On a clean $\mathrm{Cu}(111)$ metal surface, T4PT leads to the formation of a molecular honeycomb nanomesh, as shown in Fig. 1(a). The T4PT molecules are oriented along lines parallel to the [110] direction of the $\mathrm{Cu}(111)$ surface. The nanomesh has a hexagonal unit cell with a side length of $a=b=25 \pm 1 \AA$. At temperatures of $300 \mathrm{~K}$ or higher and the deposition rates of $0.1 \mathrm{ML} / \mathrm{min}$, a sufficient amount of $\mathrm{Cu}$ adatoms is available [33] to form metal-organic coordination bonds to the active nitrogen sites of the T4PT molecules, as has been observed also in other metal-organic networks on $\mathrm{Cu}(111)[3,34,35]$. The excess of $\mathrm{Cu}$ atoms is mediated by

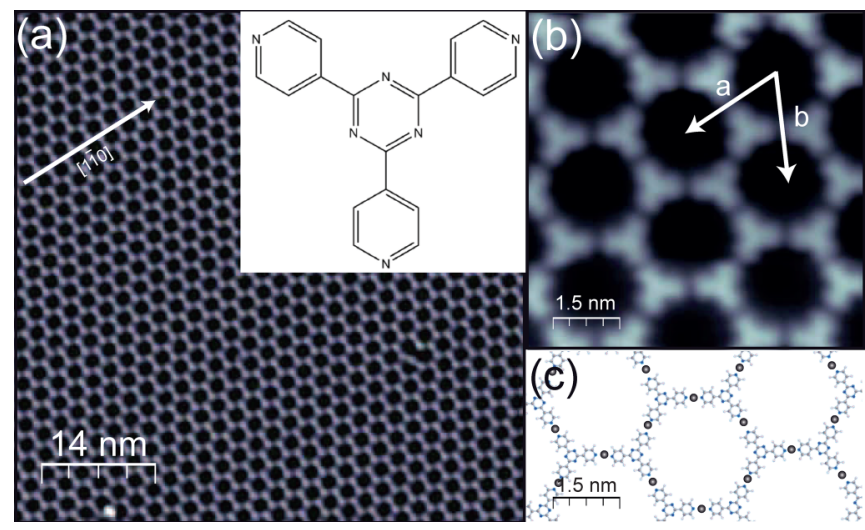

FIG. 1. (Color online) STM topography images of the Cu-T4PT nanomesh on $\mathrm{Cu}(111)$. (a) Large-scale STM topography image of the coordinated $\mathrm{Cu}-\mathrm{T} 4 \mathrm{PT}$ islands on the $\mathrm{Cu}(111)$ surface, prepared at $380 \mathrm{~K}\left(I=10 \mathrm{pA}, V_{S}=-1.0 \mathrm{~V}\right)$. Inset: Chemical structure of T4PT. (b) Zoom-in of the $\mathrm{Cu}-\mathrm{T} 4 \mathrm{PT}$ island revealing a honeycomblike structure with a twofold-coordination motif of $\mathrm{Cu}$ and T4PT $(I=10 \mathrm{pA}$, $\left.V_{S}=-0.5 \mathrm{~V}\right)$. The arrows labeled " $a$ " and " $b$ " indicate the unit-cell vectors with $a=b=25 \pm 1 \AA$ and an angle of $60.0^{\circ} \pm 1.0^{\circ}$. (c) Structure model of the $\mathrm{Cu}-\mathrm{T} 4 \mathrm{PT}$ nanomesh. 
thermal evaporation from the monoatomic steps of the $\mathrm{Cu}(111)$ surface $[35,36]$.

An enlarged high-resolution STM topography image of the $\mathrm{Cu}-\mathrm{T} 4 \mathrm{PT}$ island shows that each unit cell contains two T4PT molecules [Fig. 1(b)], such that each of the honeycomb pores is formed by six T4PT molecules. Each T4PT monomer can be now identified by its characteristic threefold symmetry.

The honeycomb nanomesh exhibits a local twofoldcoordination motif between pyridine groups of the T4PT molecules. The bonding structure is very unlikely for uncoordinated pyridine groups due to the repulsive character of the nitrogen lone-pair electrons and, therefore, suggests the presence of a bridging $\mathrm{Cu}$ adatom. Twofold coordination of $\mathrm{Cu}$ atoms with nitrogen-based end groups is known from previous works on metal-organic networks on metal surfaces [6,13-15].

In order to investigate the effect of the coordination bonding on the electronic structure [37-39], we performed scanning tunneling spectroscopy (STS) on the T4PT molecules of the $\mathrm{Cu}-\mathrm{T} 4 \mathrm{PT}$ nanomesh. Figure 2(a) shows two $d I / d V$ spectra taken at the central triazine ring of two opposing T4PT molecules of a nanomesh pore [indicated by the dots labeled "A" and "B" in the inset of Fig. 2(a)]. Both spectra exhibit a broad feature at around $0.7 \mathrm{~V}$ and a pronounced resonance at higher energies, which appears slightly shifted and with different intensity in neighboring molecules.

The distinct alternation of the two molecular types is directly resolved in differential conductance $(d I / d V)$ maps

(a)
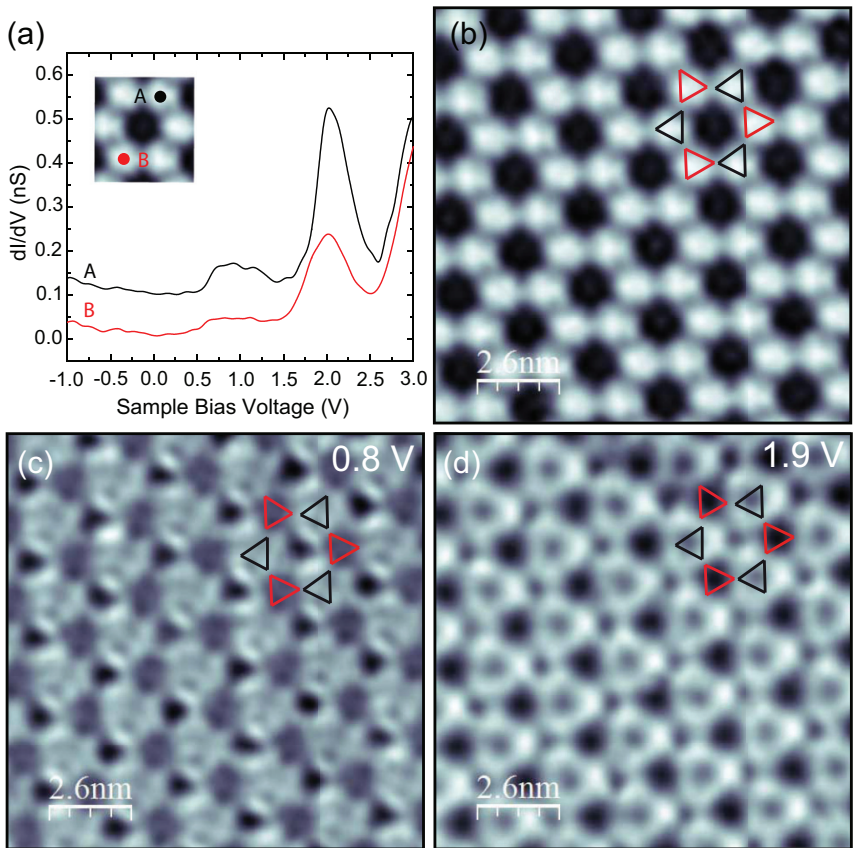

FIG. 2. (Color online) Differential conductance spectra and maps of the $\mathrm{Cu}-\mathrm{T} 4 \mathrm{PT}$ nanomesh, prepared at $380 \mathrm{~K}$. (a) Two differential conductance spectra taken at the central triazine ring of two opposing T4PT molecules (indicated by the dots labeled "A" and "B"). Curve A has been vertically offset for clarity. (b) STM topography image of the $\mathrm{Cu}-\mathrm{T} 4 \mathrm{PT}$ nanomesh and corresponding constant current $d I / d V$ maps at sample bias voltages of $0.8 \mathrm{~V}$ (c) and $1.9 \mathrm{~V}(\mathrm{~d})$, respectively $(I=0.22 \mathrm{nA})$. Triangles indicate the positions of T4PT units of types A and B.
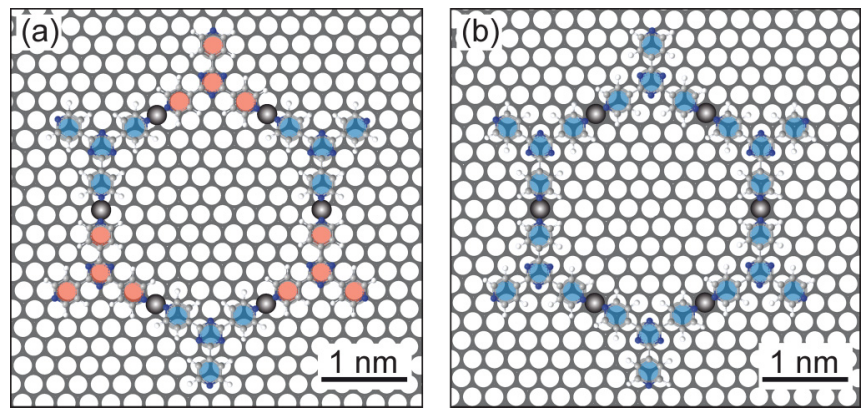

FIG. 3. (Color online) Structure model of the $\mathrm{Cu}-\mathrm{T} 4 \mathrm{PT}$ metalorganic nanomesh on the $\mathrm{Cu}(111)$ surface. The two possible positions of the coordinated $\mathrm{Cu}$ atoms are either a hollow site (adatom) (a) or a top site (surface atom) (b). According to the adsorption position of the pyridine groups, the T4PT molecules are highlighted in red [pyridine centered at top sites of the $\mathrm{Cu}(111)$ surface] or in blue [pyridine centered at hollow sites of the $\mathrm{Cu}(111)$ surface].

[Figs. 2(c) and 2(d)] [40]. At 0.8 and $1.9 \mathrm{eV}$, neighboring molecules show an alternation of high and low differential conductance signal, revealing the two electronically distinct types of T4PT molecules, here labeled as type A and type B. The different electronic structure of the molecules suggests that they are lying in different adsorption sites. Figures 3(a) and 3(b) show two possible structural models of the $\mathrm{Cu}-\mathrm{T} 4 \mathrm{PT}$ nanomesh. Both models result in equal dimensions and differ only in a lateral shift with respect to the $\mathrm{Cu}(111)$ metal surface. Under the assumption that the metal-organic nanomesh is commensurate with the surface, the distance between two neighboring $\mathrm{Cu}$ atoms in coordination sites is about $12.6 \AA$.

In Fig. 3(a), the $\mathrm{Cu}$ adatoms are at hollow sites of the $\mathrm{Cu}(111)$ surface, and the pyridine and triazine rings centered either at a top site or on a hollow site, thus defining two alternating adsorption sites of T4PT molecules in the nanomesh structure. In contrast, the model in Fig. 3(b) shows

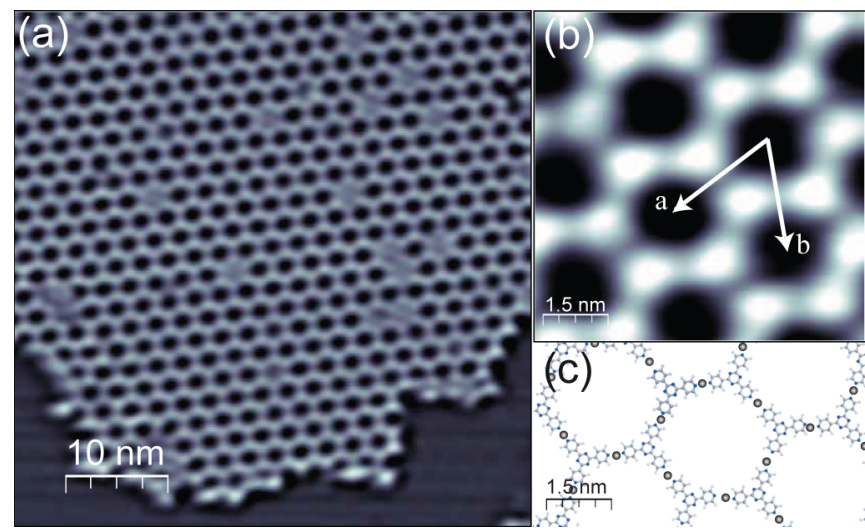

FIG. 4. (Color online) STM topography images of the $\mathrm{Cu}-\mathrm{T} 4 \mathrm{PT}$ nanomesh on $\operatorname{Ag}(111)$. (a) Large-scale STM topography image of the coordinated $\mathrm{Cu}$-T4PT islands $\left(I=20 \mathrm{pA}, V_{S}=1.0 \mathrm{eV}\right)$. (b) Zoom-in of the $\mathrm{Cu}-\mathrm{T} 4 \mathrm{PT}$ island revealing a honeycomblike structure with a twofold-coordination motif of $\mathrm{Cu}$ and T4PT $(I=0.45$ $\left.\mathrm{nA}, V_{S}=-1.5 \mathrm{eV}\right)$. The arrows labeled " $a$ " and " $b$ " indicate the unit-cell vectors with $a=b=25 \pm 1 \AA$ with an angle of $60^{\circ} \pm 1^{\circ}$. (c) Structure model of the $\mathrm{Cu}-\mathrm{T} 4 \mathrm{PT}$ nanomesh. 
$\mathrm{Cu}$ atoms at top sites of the $\mathrm{Cu}(111)$ surface, and now all T4PT molecules are centered at the same position (hollow sites) of the $\mathrm{Cu}(111)$ substrate. Since the STS data identify two different types of T4PT molecules in the $\mathrm{Cu}$-T4PT nanomesh, the correct adsorption model is the one shown in Fig. 3(a). This further agrees with the fact that single $\mathrm{Cu}$ adatoms on a $\mathrm{Cu}(111)$ surface tend to occupy hollow sites [41].

On a $\mathrm{Ag}(111)$ surface, we observe a densely packed $\mathrm{H}$-bonded network of T4PT molecules unless $\mathrm{Cu}$ atoms are codeposited along with T4PT molecules. Then, networks with the identical nanomesh structure as on $\mathrm{Cu}(111)$ are formed (see Fig. 4). The similar bonding node and unit cell as on $\mathrm{Cu}(111)$ indicate the dominant role of the metal-ligand interaction between the T4PT molecules and the $\mathrm{Cu}$ atoms, and the minor role of the underlying metal surface. Using $\operatorname{Ag}(111)$ as substrate allows us to apply the element-selective methods $\mathrm{XAS}$ and XMCD to investigate also the electronic properties of the $\mathrm{Cu}$ atoms in the network, which is not possible on top of a $\mathrm{Cu}$ substrate.

\section{B. X-ray absorption and dichroism experiments}

Having established that $\mathrm{Cu}-\mathrm{T} 4 \mathrm{PT}$ networks are formed on the two surfaces, we will now address the questions about the oxidation state of the $\mathrm{Cu}$ ions, about the presence of a magnetic moment, and about which nitrogen sites in the T4PT molecule bind to $\mathrm{Cu}$ atoms. We do so by element-specific XAS and XMCD experiments in combination with transition potential DFT simulations. This allows the assignment of peaks obtained from XAS to specific subunits of the T4PT
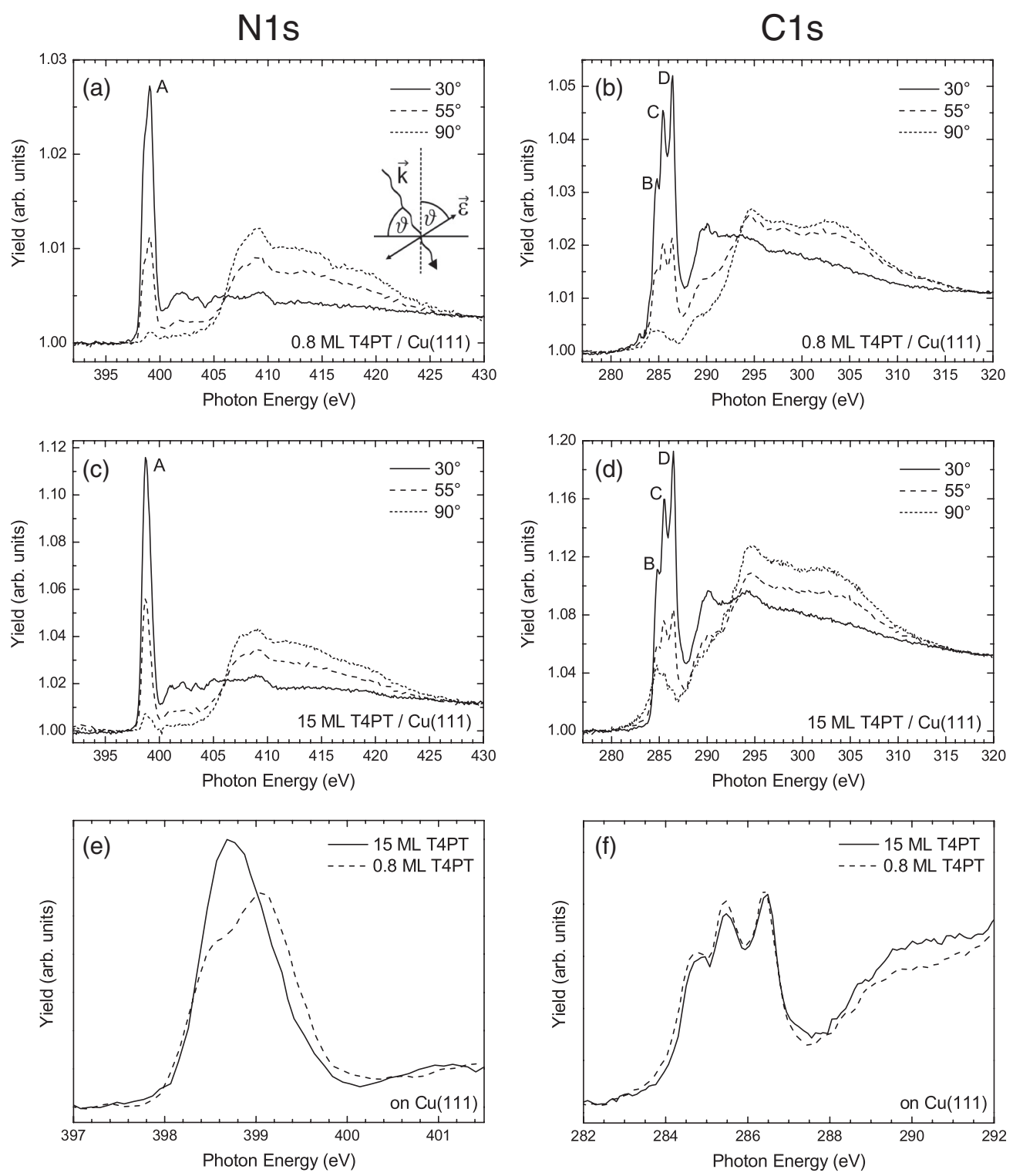

FIG. 5. (a) and (b) show polarization-resolved experimental $\mathrm{N}$ and $\mathrm{C} K$-edge XA signals for polarization angles $\vartheta=30^{\circ}$ (near grazing incidence), $\vartheta=55^{\circ}$ (magic angle), and $\vartheta=90^{\circ}$ (normal incidence) of $0.8 \mathrm{ML}$ T4PT on $\mathrm{Cu}(111)$ (submonolayer), respectively. The inset in (a) depicts the definition of the incidence angle $\vartheta$. (c) and (d) depict the polarization-resolved experimental N and C $K$-edge XA signals of $15 \mathrm{ML}$ T4PT on $\mathrm{Cu}(111)$ (multilayer). Comparison of the $\mathrm{N}$ (e) and $\mathrm{C}$ (f) $K$-edge XA signals of the 15-ML and 0.8-ML T4PT/Cu(111) samples at $\vartheta=55^{\circ}$. The spectra in (e) and (f) are scaled to match. 
molecule. Therefore, we are able to determine the coordinative character of electronic properties of different sites within T4PT molecules by studying the $\mathrm{N}$ and $\mathrm{C} K$ edges.

Figures 5(a) and 5(c) show the N $K$ edge of 0.8- and 15monolayer (ML) thin films of T4PT on $\mathrm{Cu}(111)$, respectively. While the signal of $0.8 \mathrm{ML}$ T4PT is expected to be dominated by coordinated T4PT molecules in $\mathrm{Cu}-\mathrm{T} 4 \mathrm{PT}$ networks, the spectra of the multilayer coverage of $15 \mathrm{ML}$ represent the signal of mainly uncoordinated T4PT molecules, and are used as a reference. In both cases, the XA signal consists of sharp resonances in the $\pi^{*}$ region (labeled A) centered at an energy of $399.0 \mathrm{eV}$ and broad structures in the $\sigma^{*}$ region above $406 \mathrm{eV}$ [Fig. 5(a)]. Their angle dependence is such that the intensity of peak A decreases as the angle $\vartheta$ between the surface and the $\vec{k}$ vector of the $\mathrm{x}$ rays is increasing, whereas the structure in the $\sigma^{*}$ region shows the opposite behavior. This angular dependence of the XA signal is typical for aromatic species adsorbed flat on a metal surface and, therefore, is consistent with the STM results for the submonolayer sample. It further means that also in the case of the 15-ML T4PT/Cu(111) sample the molecular plane of the T4PT molecules is orientated parallel to the metal surface.

The main difference between the spectra of the 0.8- and the 15-ML samples is the line shape of peak A. Figure 5(e) shows a comparison of this peak in the two samples, scaled onto each other for comparison of the line shape. Peak A of the multilayer sample consists of a single peak at $398.7 \mathrm{eV}$. Therefore, both nitrogen species of the T4PT, the ones from the pyridine as well as from the triazine groups, overlap in their contribution to this peak. Peak A of the 0.8-ML T4PT/Cu(111) sample shows a double-peak structure with one contribution at $398.7 \mathrm{eV}$ as in the multilayer sample, and one additional peak at $399.1 \mathrm{eV}$. This finding suggests that the $\mathrm{Cu}$ adatoms bond solely to the pyridine terminations of the T4PT molecule, leaving the triazine unit uncoordinated. The coordination of $\mathrm{Cu}$ atoms to the $\mathrm{N}$ atoms in the triazine rings is probably sterically prohibited by the presence of the neighboring $\mathrm{H}$ atoms of the pyridine groups. We will sustain this argument later in this paper by means of theoretical simulations.

To resolve a possible electronic interaction and charge transfer of the molecular backbone with the substrate, we now examine the $\mathrm{C}$ edges. The $\pi^{*}$ region of the $\mathrm{C} K$-edge XA signal of the $0.8-\mathrm{ML}$ T4PT/Cu(111) sample shows three peaks, labeled B-D in Fig. 5(b). Peak B is centered at an energy of $284.8 \mathrm{eV}$, followed by peaks $\mathrm{C}$ and $\mathrm{D}$ at energies of 285.5 and $286.4 \mathrm{eV}$, respectively. These three peaks in the $\pi^{*}$ region are followed by a broad structure in the $\sigma^{*}$ region above $289 \mathrm{eV}$. Similar to the $\mathrm{N} K$-edge XA signal, also the resonances in the carbon spectra exhibit a clear angular dependence. Peaks B-D decrease with increasing angle $\vartheta$, in contrast to the $\sigma^{*}$ region intensity, which increases. Again, this finding agrees with the fact that the T4PT molecules are
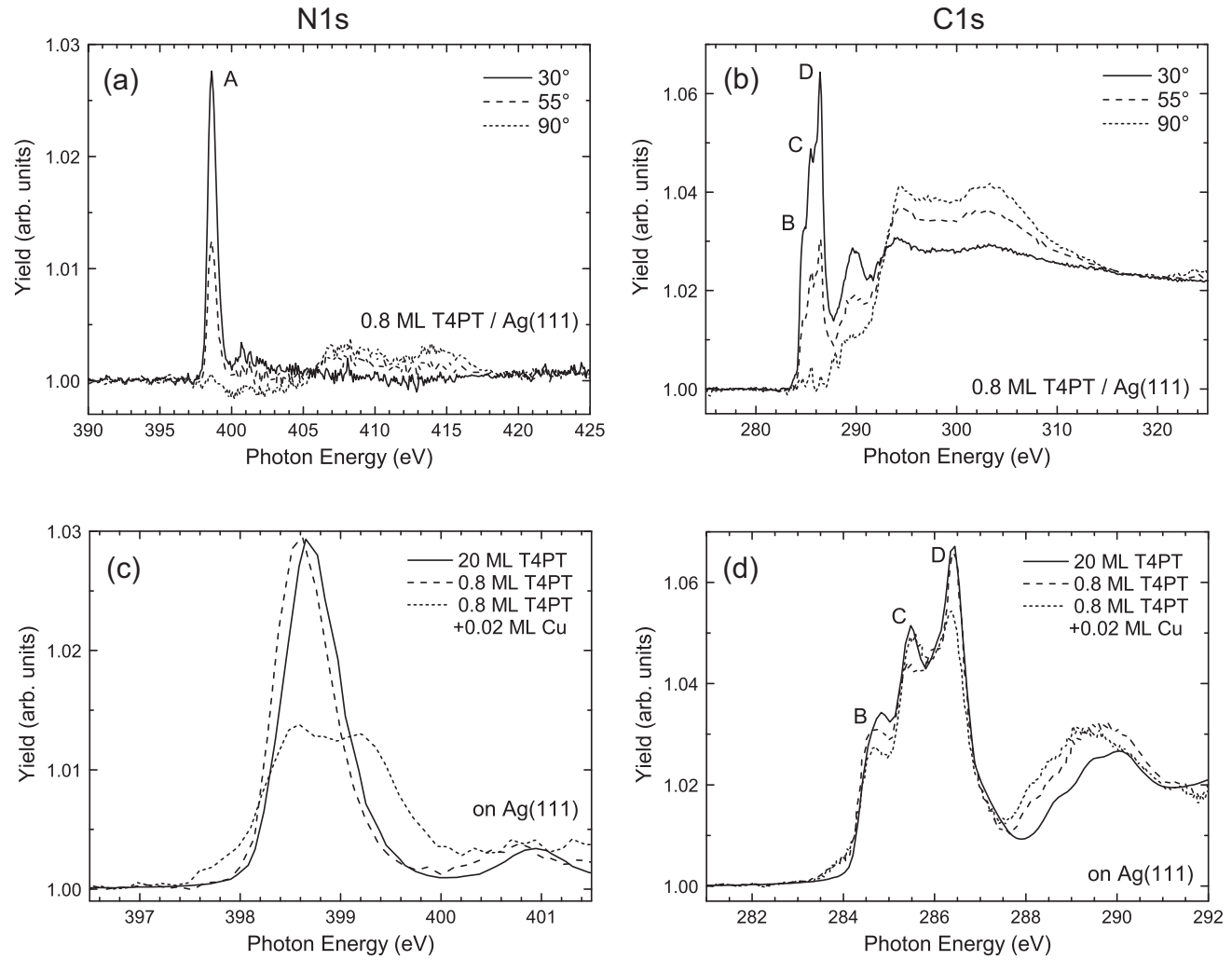

FIG. 6. (a) and (b) show the polarization-resolved experimental $\mathrm{N}$ and $\mathrm{C} K$-edge XA signals for polarization angles $\vartheta=30^{\circ}$ (near grazing incidence), $\vartheta=55^{\circ}$ (magic angle), and $\vartheta=90^{\circ}$ (normal incidence) of an 0.8-ML T4PT/Ag(111) sample. (c) N $K$-edge XA signals for a polarization angle $\vartheta=30^{\circ}$ of the 20-ML T4PT/Ag(111) sample, the 0.8-ML T4PT/Ag(111) sample, and of the 0.8-ML T4PT/Ag(111) sample after deposition of $0.02 \mathrm{ML} \mathrm{Cu}$, respectively. The spectrum of the multilayer is scaled for comparison. (d) $\mathrm{C} K$-edge XA signals for a polarization angle $\vartheta=30^{\circ}$ of the 20-ML T4PT/Ag(111) sample, the 0.8-ML T4PT/Ag(111) sample, and of the 0.8-ML T4PT/Ag(111) sample after deposition of $0.02 \mathrm{ML} \mathrm{Cu}$, respectively. The spectrum of the multilayer is scaled for comparison. 
adsorbed flat with their molecular plane parallel to the surface plane.

Very similar C $1 s$ spectra are obtained from the 15-ML sample [Fig. 5(d)]. The $\pi^{*}$ region of the C $K$-edge XA signal is almost identical to the one of the $0.8-\mathrm{ML}$ T4PT/Cu(111) sample, as can be seen in Fig. 5(f), which shows a zoom-in of the $\pi^{*}$ region. Here, the spectra are also scaled onto each other for a better comparison of the line shape. All peaks (B-D) in the $\pi^{*}$ region are visible in both spectra. Also, the peak positions are nearly the same. Hence, the formation of a metal-ligand bond has a minor impact on the carbon species in terms of their electronic structure.

Since on the $\operatorname{Ag}(111)$ surface analogous $\mathrm{Cu}-\mathrm{T} 4 \mathrm{PT}$ networks can be formed by deposition of $\mathrm{Cu}$ atoms on a submonolayer of T4PT, this system can be used to obtain information on the electronic properties of the $\mathrm{Cu}$ atoms. We first show in Fig. 6 that the T4PT molecules exhibit a similar electronic structure on $\mathrm{Ag}(111)$ and $\mathrm{Cu}(111)$. Figure 6(a) shows the polarization-resolved $\mathrm{N} K$-edge XA signals of 0.8 ML of T4PT/Ag(111) (without $\mathrm{Cu}$ ) for different incident angles. The corresponding $\mathrm{C} K$-edge spectra are presented in Fig. 6(b). A detailed comparison of the $\mathrm{N}$ and $\mathrm{C} K$ edges of 0.8 and $20 \mathrm{ML}$ T4PT on $\mathrm{Ag}(111)$ in the $\pi^{*}$ region is shown in Figs. 6(c) and 6(d), respectively. All the spectra of uncoordinated T4PT molecules in a submonolayer on $\mathrm{Ag}(111)$ are very similar to the ones of a multilayer on $\operatorname{Ag}(111)$, and also to the multilayer of T4PT on $\mathrm{Cu}(111)$ [cf. Figs. 5(c) and 5(d)]. The similarity between the spectra of the submonolayer on $\mathrm{Ag}(111)$ and a multilayer of T4PT points towards a minor interaction of the T4PT molecules with the $\mathrm{Ag}(111)$ metal surface.

Figure 6(c) also includes the comparison with the $\mathrm{N}$ $K$-edge XA signal of the $0.8-\mathrm{ML}$ T4PT/Ag(111) sample after subsequent deposition of $0.02 \mathrm{ML}$ of $\mathrm{Cu}$ atoms. The coordination of the pyridine nitrogen atoms with the $\mathrm{Cu}$ again leads to the appearance of a double-peak feature in the $\pi^{*}$ region, similar as in the $0.8-\mathrm{ML} \mathrm{T} 4 \mathrm{PT} / \mathrm{Cu}(111)$ sample. We take this as a fingerprint of the formation of a coordination bond. Once all T4PT molecules are coordinated, the increase of $\mathrm{Cu}$ atoms on the $\mathrm{Ag}(111)$ surface does not lead to a further increase of the intensity of the double-peak feature at $399.2 \mathrm{eV}$ in the $\pi^{*}$ region (not shown here). The change of the $\mathrm{C} K$-edge $\mathrm{XA}$ signal upon deposition of $\mathrm{Cu}$ atoms is rather small as can be seen in Fig. 6(d).

The findings discussed above are in line with results on the T4PT samples prepared on a $\mathrm{Cu}(111)$ metal surface. Hence, the $\mathrm{Cu}-\mathrm{T} 4 \mathrm{PT}$ metal-organic networks on $\mathrm{Ag}(111)$ show the same spectral features as on $\mathrm{Cu}(111)$, evidencing their resemblance in terms of their structural and electronic properties.

\section{Calculations of $x$-ray absorption spectra}

We will now show that the changes observed in the $\mathrm{N}$ $K$-edge absorption spectra are indeed due to formation of a bond between the pyridine $\mathrm{N}$ atoms and $\mathrm{Cu}$ by assigning the different contributions to the spectra. The theoretical studies consider the free 2,4,6-tris(4-pyridine)-1,3,5-triazine (T4PT) molecule as a building unit of the metal-organic network. T4PT contains two types of nitrogen, N1, N2, and four types of carbon atoms, $\mathrm{C} 1$ to $\mathrm{C} 4$, which are not equivalent by (a)

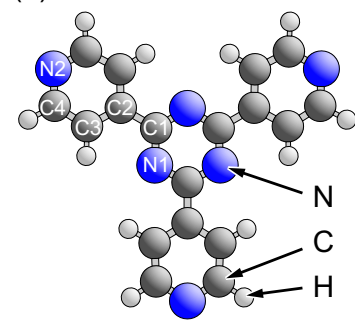

(b)

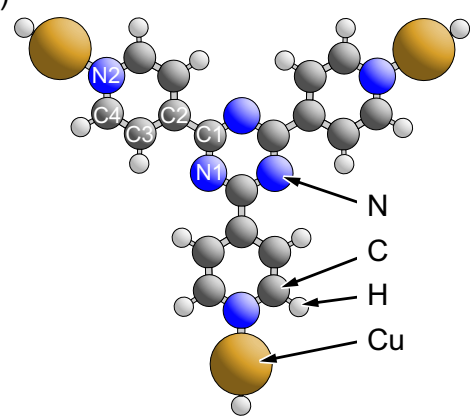

FIG. 7. (Color online) Optimized geometric structures of (a) the free T4PT molecule, (b) the T4PT- $(\mathrm{CuH})_{3}$ model cluster. Atoms are shown by balls of different radii and color and labeled accordingly. Symmetry nonequivalent atoms considered for core excitations in the calculations, nitrogen $\mathrm{N} 1, \mathrm{~N} 2$, carbon $\mathrm{C} 1-\mathrm{C} 4$, are denoted in the figures.

symmetry [see Fig. 7(a)]. Here, we simulate the local coupling with neighboring T4PT units in coordination network with $\mathrm{Cu}$ atoms by adding $\mathrm{Cu}$ near the outer $\mathrm{N} 2$ atoms and saturating the dangling $\mathrm{Cu}$ bonds to T4PT neighbors by hydrogen which, altogether, yields a T4PT- $(\mathrm{CuH})_{3}$ model cluster as shown in Fig. 7(b).

The calculations are based on relaxed structures of the free T4PT molecule and the T4PT- $(\mathrm{CuH})_{3}$ cluster depicted in Figs. 7(a) and 7(b). The resulting bond distances for both relaxed structures are given in Table I. The coordination bond is described properly in our calculations because the distance between the $\mathrm{Cu}$ atom and the outer pyridine nitrogen N2 (see Fig. 7) is $1.945 \AA$, in agreement with previous calculations of networks of anthracenedicarbonitrile molecules and $\mathrm{Cu}$ atoms that had yielded a value of $1.99 \AA$ along the $\mathrm{Cu}-\mathrm{N}$ vector [34]. It lies within the experimental $\mathrm{Cu}-\mathrm{N}$ distance of $\approx 2 \AA$ estimated from the structure model obtained from the STM results (Fig. 1). We further note that the formation of a metal-ligand bond between the pyridine nitrogens (N2) and the $\mathrm{Cu}$ atoms has little effect on the T4PT intramolecular bond lengths.

The interaction of $\mathrm{Cu}$ atoms with the T4PT molecules should lead to essential changes in the $\mathrm{N} K$-edge XA spectrum of the T4PT nitrogen species (N1 and N2). The comparison of the experimental results with the theoretical XA spectra allows the assignment of the corresponding peaks and also the

TABLE I. The optimized bond lengths of the free T4PT molecule and the T4PT- $-(\mathrm{CuH})_{3}$ cluster.

\begin{tabular}{lcc}
\hline \hline & Bond distances $(\AA)$ & \\
\hline & $\mathrm{T} 4 \mathrm{PT}$ & $\mathrm{T} 4 \mathrm{PT}-\mathrm{Cu}_{3} \mathrm{H}_{3}$ \\
\hline $\mathrm{Cu}-\mathrm{N} 2$ & & 1.945 \\
$\mathrm{~N} 1-\mathrm{C} 1$ & 1.353 & 1.355 \\
$\mathrm{~N} 2-\mathrm{C} 4$ & 1.353 & 1.366 \\
$\mathrm{C} 1-\mathrm{C} 2$ & 1.500 & 1.495 \\
$\mathrm{C} 2-\mathrm{C} 3$ & 1.408 & 1.412 \\
$\mathrm{C} 3-\mathrm{C} 4$ & 1.404 & 1.395 \\
$\mathrm{Cu}-\mathrm{H}$ & & 1.505 \\
\hline \hline
\end{tabular}



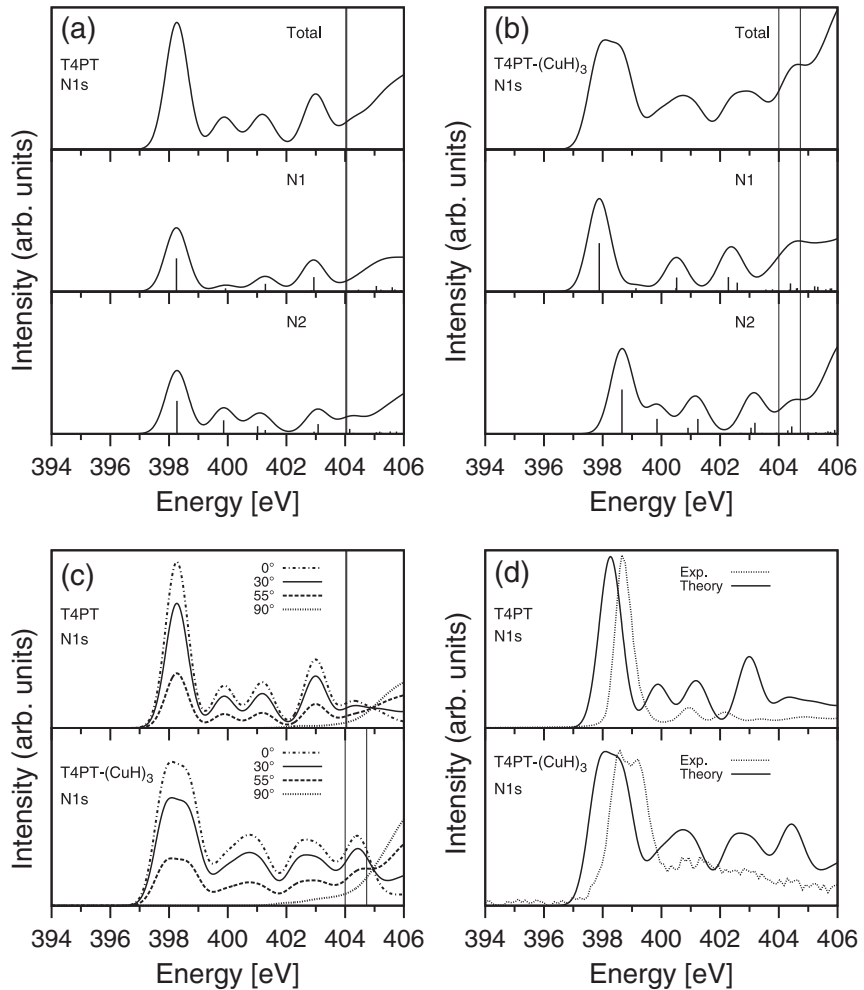

FIG. 8. (a) Theoretical N $K$-edge XA signals for a free T4PT molecule; total spectrum (upper panel) and decomposition into contributions of the nitrogen centers N1 and N2 (middle and bottom panels). (b) Theoretical N $K$-edge XA spectra for the T4PT- $(\mathrm{CuH})_{3}$ cluster as in (a). The small vertical strokes in (a) and (b) show the discrete excitations obtained in the calculations with their lengths denoting the excitation probabilities. (c) Theoretical polarizationresolved N $K$-edge XA spectra for polarization angles $\vartheta=0^{\circ}, 30^{\circ}$, $55^{\circ}, 90^{\circ}$ of free T4PT (top panel) and of the T4PT- $(\mathrm{CuH})_{3}$ cluster (bottom panel). The vertical lines close to $404 \mathrm{eV}$ in (a), (b), and (c) indicate the ionization thresholds. (d) $\mathrm{N} K$-edge XA spectra at a polarization angle $\vartheta=30^{\circ}$, comparison for free T4PT (theory) with data from 20-ML T4PT/Ag(111) (experiment) (upper panel) and for T4PT- $(\mathrm{CuH})_{3}$ (theory) with data from a $\mathrm{Ag}(111)$ sample with $0.02 \mathrm{ML}$ of $\mathrm{Cu}$ and $0.8 \mathrm{ML}$ of T4PT.

analysis of the metal-ligand bond formation. Figure 8(a) shows the total (polarization-angle integrated) theoretical spectrum of the free T4PT molecule. Both nitrogen species N1 and $\mathrm{N} 2$ contribute to the spectrum with a peak in the $\pi^{*}$ region located at $398.3 \mathrm{eV}$. The vertical line close to $404 \mathrm{eV}$ marks the ionization threshold. The experimentally observed peak A in the $\pi^{*}$ region of the thicker films at the energy of $398.7 \mathrm{eV}$ is the sum of the two contributions originating from the triazine and pyridine nitrogen species N1 and N2. Hence, both contributions can not be distinguished experimentally [see Figs. 5(c) and 6(a)]. This finding implies that the chemical environment for the two nitrogen species is very similar in the case of the uncoordinated T4PT molecule.

Figure 8 (b) shows the theoretical N $K$-edge XA spectrum of the T4PT- $(\mathrm{CuH})_{3}$ cluster. In contrast to the spectra of the uncoordinated T4PT (free molecule), the energetic positions of the spectral contributions of nitrogen species N1 and N2 are shifted with respect to each other. The contribution of the triazine nitrogen N1 is located at an energy of $397.9 \mathrm{eV}$, shifted to lower binding energies, and the pyridine nitrogen N2 is located at an energy of $398.6 \mathrm{eV}$, shifted to higher binding energies with respect to the uncoordinated case. Figure 8(c) shows the theoretical polarization-resolved $\mathrm{N} K$-edge XA spectra of both the free T4PT molecule and the T4PT- $(\mathrm{CuH})_{3}$ cluster. The angle dependence of the spectral features of the $\mathrm{N}$ $K$-edge found experimentally is reproduced in the simulations [see Fig. 5(a)]. Thus, the flat adsorption geometry of the T4PT molecules is further corroborated.

Figure 8(d) shows a detailed comparison of the results of our calculation and the experimental data for the polarization angle $\vartheta=30^{\circ}$. Apart from a global energy shift of about $0.4 \mathrm{eV}$, the calculated N $K$-edge XA spectra of the free T4PT molecule and the T4PT- $(\mathrm{CuH})_{3}$ cluster are in very good agreement with the experimental data, confirming the coordination of the pyridine nitrogen atoms N2. Hence, the coordination of these nitrogen atoms with the $\mathrm{Cu}$ atoms leads to a shift of the XAS contribution of the pyridine nitrogens, while the peak from triazine nitrogens remains at an almost constant energy, indicating that the triazine units stay uncoordinated.

The energetic shift of the pyridine N $K$-edge XA contribution in the $\pi^{*}$ region is attributed to metal-ligand interaction with the $\mathrm{Cu}$ adatoms. A Bader charge analysis reveals that the T4PT molecule in the T4PT- $(\mathrm{CuH})_{3}$ cluster gains a total charge of $\rho=0.03 e^{-}$as a result of the formation of metal-ligand bonds. This almost insignificant charge transfer is in line with recently performed DFT simulations on a very similar metal-organic network on $\mathrm{Cu}(111)$ [14]. Despite the rather small value, the energetic shift of the peak position may be related to the metal-ligand bond interaction. The shift to higher photon energies could either come from a shift of the LUMO $+n$ to higher energy, or a reduction of the electronic density at the position of the respective nitrogen atoms. The latter would be consistent with the intuitive picture that the nonbonding electrons are pulled further away from the nitrogen core by the additional coordination bond to the $\mathrm{Cu}$ adatoms.

The experimental and simulated XA C $K$-edge spectra are shown in Fig. 9. Figure 9(a) shows the total (polarizationangle integrated) theoretical spectrum of the free gas-phase T4PT molecule (upper panel) and its decomposition into contributions of the different $\mathrm{C} 1, \mathrm{C} 2, \mathrm{C} 3$, and $\mathrm{C} 4$ excitation centers. The broad spectral feature in the $\pi^{*}$ region of the C $K$ edge consists of several contributions of the different carbon species. Species C1 to C3 contribute each with two components, which are slightly shifted to higher binding energies by going from $\mathrm{C} 1$ to $\mathrm{C} 3$. The carbon species $\mathrm{C} 4$ has a single peak in the $\pi^{*}$ region located at an energy of $286.3 \mathrm{eV}$. The C1- to C4-derived peak shifts can be understood in terms of the different chemical environment. The carbon species $\mathrm{C} 1$ faces two nitrogen neighbors. Since the nitrogen species is rather electronegative, the charge density of the carbon atom is slightly displaced towards the neighboring nitrogen atoms, implying a slightly positive charge. Therefore, the atomic core-level energy is shifted to higher energies.

Figure 9(b) shows the theoretical $\mathrm{C} K$-edge XA spectrum of the T4PT- $(\mathrm{CuH})_{3}$ cluster. The overall shape of the spectrum is very similar to the one of the free T4PT molecule shown in Fig. 9(a) indicating that the formation of the metal-ligand bond 

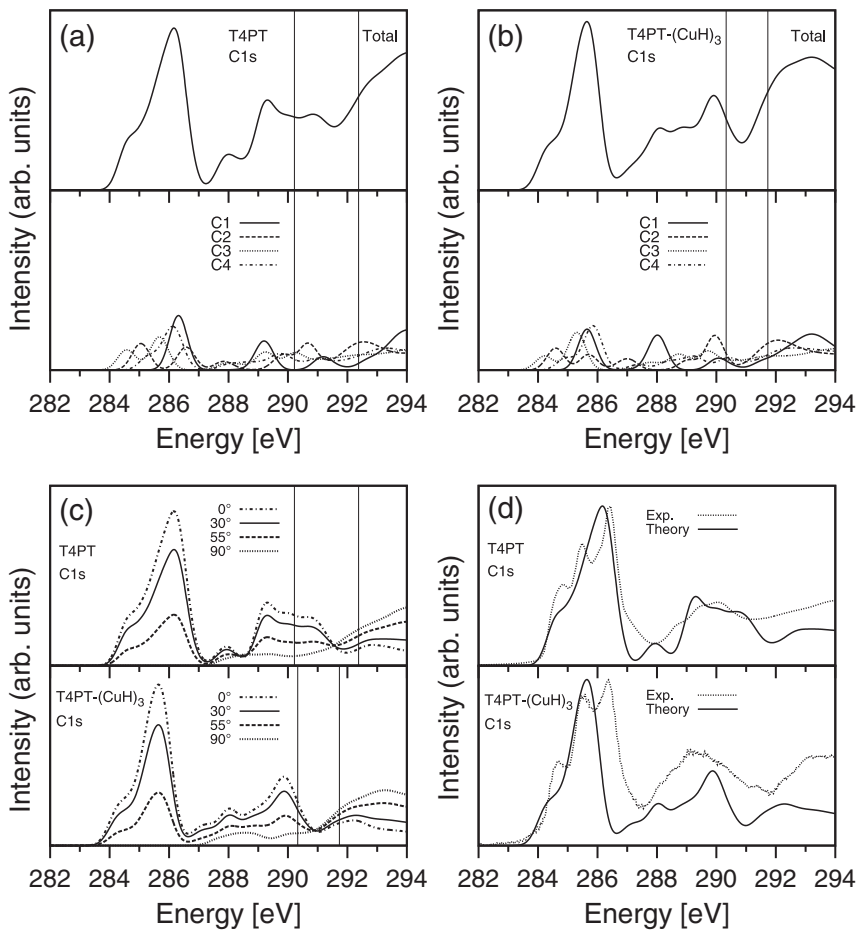

FIG. 9. (a) Theoretical C $K$-edge XA spectra for a free T4PT molecule; total spectrum (upper panel) and decomposition into contributions of the nitrogen centers N1 and N2 (bottom panel). (b) Theoretical C $K$-edge XA spectra for the T4PT- $(\mathrm{CuH})_{3}$ cluster as in (a). (c) Theoretical polarization-resolved C $K$-edge XA spectra for polarization angles $\vartheta=0^{\circ}, 30^{\circ}, 55^{\circ}, 90^{\circ}$ of free T4PT (top panel) and of the T4PT- $(\mathrm{CuH})_{3}$ cluster (bottom panel). The vertical lines near 290 and $292 \mathrm{eV}$ in (a), (b), and (c) indicate the range of ionization thresholds for the different carbon species. (d) C $K$-edge XA spectra at a polarization angle $\vartheta=30^{\circ}$, comparison for free T4PT (theory) with data from $20 \mathrm{ML}$ T4PT/Ag(111) (experiment) (upper panel) and for T4PT- $(\mathrm{CuH})_{3}$ (theory) with data from a $\mathrm{Ag}(111)$ sample with $0.02 \mathrm{ML}$ of $\mathrm{Cu}$ and $0.8 \mathrm{ML}$ of T4PT.

has only a minor effect on the electronic structure of the carbon species, as suggested by our experiments. The intensities and energetic positions of the peaks stemming from the $\mathrm{C} 1$ to $\mathrm{C} 4$ carbon species are all slightly different as can be seen in the decomposition of the total spectrum into the individual contributions shown in the bottom panel of Fig. 9(b).

While Fig. 9(c) shows theoretical polarization-resolved C $K$-edge XA spectra of both the free T4PT molecule and the T4PT- $(\mathrm{CuH})_{3}$ cluster, Fig. 9(d) compares experimental and theoretical spectra at a polarization angle $\vartheta=30^{\circ}$. In case of the free T4PT molecule (upper panel), the theoretical $\mathrm{C}$ $K$-edge XA spectrum generally reproduces the experimental data. The intensities of the single peaks originating from the excitations at the carbon centers $\mathrm{C} 1$ to $\mathrm{C} 4$ are larger compared to those of the theoretical XA spectrum. The lower panel of Fig. 9(d) compares experimental and the theoretical C $K$ XA spectra of the T4PT- $(\mathrm{CuH})_{3}$ cluster. The theoretical spectrum reproduces the overall shape of the experimental XA spectrum, but in contrast to the results for the free T4PT molecule, there is an additional peak in the experimental spectrum, which is not visible in the simulation. Apparently, the intensity of some of the peaks in the theoretical spectra seems to be underestimated, possibly due to missing electronic coupling to the substrate in the model cluster.

\section{Magnetic properties of the $\mathrm{Cu}$ atoms}

In order to obtain information about the magnetic properties and the oxidation state of the $\mathrm{Cu}$ atoms inside the $\mathrm{Cu}$-T4PT metal-organic network, we carried out XAS measurements at the $\mathrm{Cu} L_{2,3}$ edges. Figure 10 (a) shows the $\mathrm{Cu} L_{2,3} \mathrm{XA}$ signal of a $0.8-\mathrm{ML}$ T4PT/Ag(111) sample after deposition of $0.02 \mathrm{ML}$ of $\mathrm{Cu}$ atoms (black line) and of $0.1 \mathrm{ML}$ of $\mathrm{Cu}$ atoms on a clean $\mathrm{Ag}(111)$ metal surface as a reference (red line) measured at $\vartheta=30^{\circ}$ grazing incidence at $T=10 \mathrm{~K}$ (the latter scaled by a factor of 0.23 for better comparison). The $\mathrm{Cu} L_{2,3}$ XA spectrum of $\mathrm{Cu}-\mathrm{T} 4 \mathrm{PT}$ is very similar to $\mathrm{Cu}$ metal spectra $[42,43]$. The steplike feature at the $\mathrm{Cu} L_{2,3}$ edges in Fig. 10(a) reflects the onset of transitions into unoccupied $s p$ states. Comparison to the reference sample of $0.1 \mathrm{ML}$ of $\mathrm{Cu}$ atoms on the clean $\mathrm{Ag}(111)$ sample (red line) without T4PT molecules shows indeed no difference in line shape, nor in energy. Both $\mathrm{Cu}$ absorption signals do not show any appreciable peak indicative of unoccupied $d$ states, just the
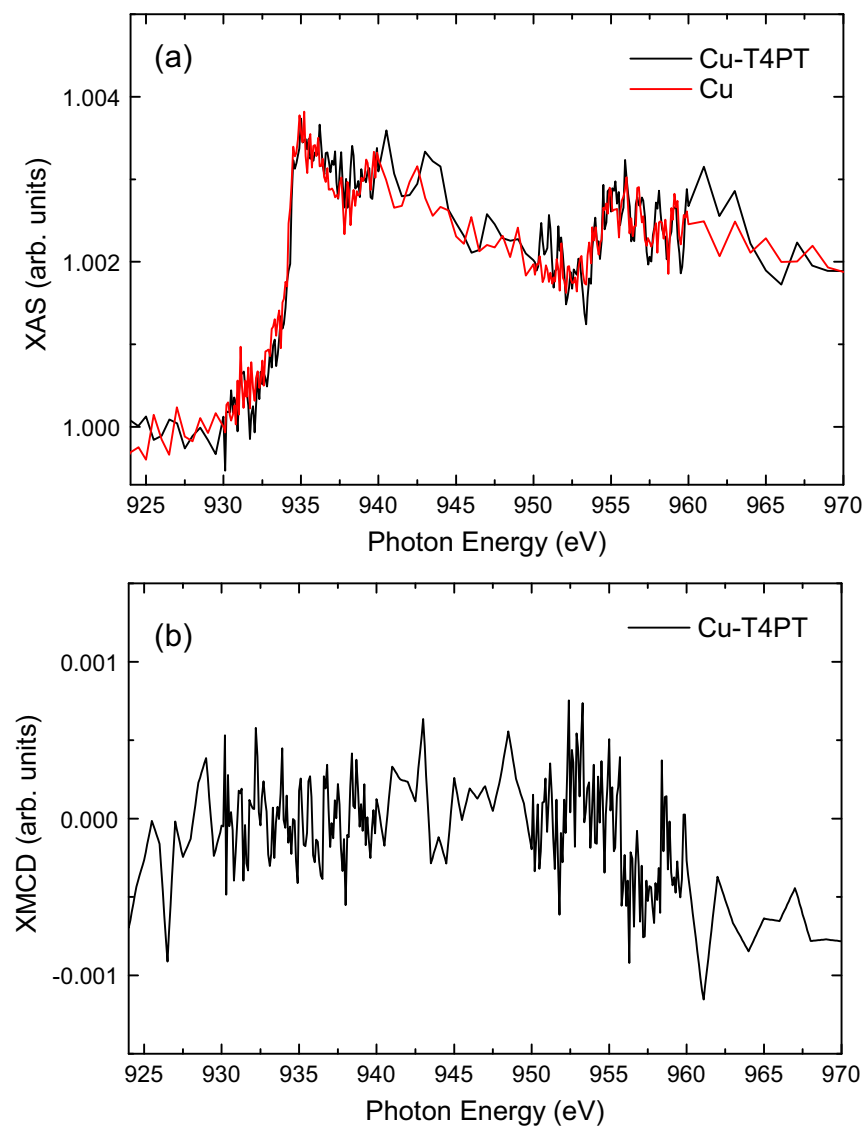

FIG. 10. (Color online) (a) $\mathrm{Cu} L_{2,3} \mathrm{XAS}$ spectra of $0.8 \mathrm{ML}$ of T4PT on $\mathrm{Ag}(111)$ after deposition of $0.02 \mathrm{ML}$ of $\mathrm{Cu}$ (black line) and of $0.1 \mathrm{ML}$ of $\mathrm{Cu}$ atoms on $\mathrm{Ag}(111)$ without molecules (red line) measured at $\vartheta=30^{\circ}$ grazing incidence at $T=10 \mathrm{~K}$. The latter is scaled by a factor of 0.23 . (b) $\mathrm{Cu} L_{2,3}$ XMCD spectra of $0.8 \mathrm{ML}$ of T4PT on $\mathrm{Ag}(111)$ after deposition of $0.02 \mathrm{ML}$ of $\mathrm{Cu}$ measured at $30^{\circ}$ grazing incidence at $T=10 \mathrm{~K}$ and $B=5 \mathrm{~T}$. 
edge jump. The absence of any $d$-derived peak evidences that $\mathrm{Cu}$ atoms are in a $d^{10}$ state.

Figure 10(b) shows the $\mathrm{Cu} L_{2,3}$ XMCD difference signal of $0.8 \mathrm{ML}$ of T4PT on $\mathrm{Ag}(111)$ after deposition of $0.02 \mathrm{ML}$ of $\mathrm{Cu}$ atoms measured at $30^{\circ}$ grazing incidence at $T=10 \mathrm{~K}$ in a magnetic field of $5 \mathrm{~T}$. Within the noise level, there is no $\mathrm{XMCD}$ signal. This fits to the conclusion that the $\mathrm{Cu}$ atoms within the $\mathrm{Cu}$-T4PT metal-organic network adopt a $d^{10}$ state and have no magnetic moment.

\section{CONCLUSIONS}

The present combined XAS, STM, and DFT study investigated structural and electronic properties of the $\mathrm{Cu}-$ T4PT metal-organic network on $\mathrm{Ag}(111)$ and $\mathrm{Cu}(111)$ crystalline surfaces. Due to dominant metal-ligand interactions, metal-organic networks are found to form honeycomb lattices with similar lattice dimensions on both metal surfaces. The XA signals of the $\mathrm{Cu}-\mathrm{T} 4 \mathrm{PT} / \mathrm{Cu}(111)$ and $\mathrm{Cu}-\mathrm{T} 4 \mathrm{PT} / \mathrm{Ag}(111)$ sample are also very similar, which confirms the minor role of the underlying metal surface on structural and electronic properties of the metal-organic network. Therefore, the present $\mathrm{Cu}$-T4PT metal-organic network is a promising candidate for surface pattern formation and functionalization. In addition, our DFT simulations allow the assignment of the individual XAS peaks. The changes of the XA signals of the uncoordinated and $\mathrm{Cu}$-coordinated T4PT molecules are attributed to metal-ligand bonds between the active nitrogen species of the pyridine subunits and the $\mathrm{Cu}$ adatoms, while the triazine unit remains uncoordinated. The magnetic properties of the coordinated $\mathrm{Cu}$ atoms were investigated in terms of XMCD revealing that the $\mathrm{Cu}$ atoms have a $d^{10}$ state and therefore no magnetic moment.

\section{ACKNOWLEDGMENT}

Funding by the DFG ( $\mathrm{Sfb} 658$ and Grant No. FR2726/1) as well as the Focus Area "Functional Materials at the Nanoscale" of the Freie Universität Berlin is gratefully acknowledged.
[1] S. Stepanow, N. Lin, D. Payer, U. Schlickum, F. Klappenberger, G. Zoppellaro, M. Ruben, H. Brune, J. V. Barth, and K. Kern, Angew. Chem., Int. Ed. 46, 710 (2007).

[2] U. Schlickum, R. Decker, F. Klappenberger, G. Zoppellaro, S. Klyatskaya, M. Ruben, I. Silanes, A. Arnau, K. Kern, H. Brune, and J. V. Barth, Nano Lett. 7, 3813 (2007).

[3] J. Björk, M. Matena, M. S. Dyer, M. Enache, J. Lobo-Checa, L. H. Gade, T. A. Jung, M. Stöhr, and M. Persson, Phys. Chem. Chem. Phys. 12, 8815 (2010).

[4] M. Marschall, J. Reichert, A. Weber-Bargioni, K. Seufert, W. Auwärter, S. Klyatskaya, G. Zoppellaro, M. Ruben, and J. V. Barth, Nat. Chem. 2, 131 (2010).

[5] J. V. Barth, Surf. Sci. 603, 1533 (2009).

[6] S. L. Tait, A. Langner, N. Lin, S. Stepanow, C. Rajadurai, M. Ruben, and K. Kern, J. Phys. Chem. C 111, 10982 (2007).

[7] T. Samuely, S.-X. Liu, M. Haas, S. Decurtins, T. A. Jung, and M. Stöhr, J. Phys. Chem. C 113, 19373 (2009).

[8] M. Pivetta, G. E. Pacchioni, U. Schlickum, J. V. Barth, and H. Brune, Phys. Rev. Lett. 110, 086102 (2013).

[9] F. Klappenberger, D. Kühne, W. Krenner, I. Silanes, A. Arnau, F. J. García de Abajo, S. Klyatskaya, M. Ruben, and J. V. Barth, Nano Lett. 9, 3509 (2009).

[10] P. Gambardella, S. Stepanow, A. Dmitriev, J. Honolka, F. M. F. de Groot, M. Lingenfelder, S. S. Gupta, D. D. Sarma, P. Bencok, S. Stanescu, S. Clair, S. Pons, N. Lin, A. P. Seitsonen, H. Brune, J. V. Barth, and K. Kern, Nat. Mater. 8, 189 (2009).

[11] N. Abdurakhmanova, T.-C. Tseng, A. Langner, C. S. Kley, V. Sessi, S. Stepanow, and K. Kern, Phys. Rev. Lett. 110, 027202 (2013).

[12] T. R. Umbach, M. Bernien, C. F. Hermanns, A. Krüger, V. Sessi, I. Fernandez-Torrente, P. Stoll, J. I. Pascual, K. J. Franke, and W. Kuch, Phys. Rev. Lett. 109, 267207 (2012).

[13] Z. Shi, J. Liu, T. Lin, F. Xia, P. N. Liu, and N. Lin, J. Am. Chem. Soc. 133, 6150 (2011).

[14] W. Wang, X. Shi, S. Wang, J. Liu, M. A. Van Hove, P. N. Liu, R.-Q. Zhang, and N. Lin, Phys. Rev. Lett. 110, 046802 (2013).
[15] J. Liu, T. Lin, Z. Shi, F. Xia, L. Dong, P. N. Liu, and N. Lin, J. Am. Chem. Soc. 133, 18760 (2011).

[16] M.-X. Li, Z.-X. Miao, M. Shao, S.-W. Liang, and S.-R. Zhu, Inorg. Chem. 47, 4481 (2008).

[17] K. Hermann and L. G. M. Pettersson, STOBE software package, see also http://www.fhi-berlin.mpg.de/KHsoftware/StoBe/

[18] B. Hammer, L. B. Hansen, and J. K. Nørskov, Phys. Rev. B 59, 7413 (1999).

[19] J. P. Perdew, K. Burke, and M. Ernzerhof, Phys. Rev. Lett. 77, 3865 (1996).

[20] J. Stöhr, NEXAFS Spectroscopy (Springer, Berlin, 1992).

[21] See Supplemental Material at http://link.aps.org/supplemental/ 10.1103/PhysRevB.89.235409 for additional computational details of the simulations of $\mathrm{x}$-ray absorption spectra and calculated Bader charges.

[22] L. Triguero, L. G. M. Pettersson, and H. Ågren, Phys. Rev. B 58, 8097 (1998).

[23] H. Ågren, V. Carravetta, O. Vahtras, and L. G. M. Pettersson, Chem. Phys. Lett. 222, 75 (1994).

[24] J. C. Slater and K. H. Johnson, Phys. Rev. B 5, 844 (1972).

[25] O. Takahashi and L. G. M. Pettersson, J. Chem. Phys. 121, 10339 (2004).

[26] M. Leetmaa, M. P. Ljungberg, A. Lyubartsev, A. Nilsson, and L. G. M. Pettersson, J. Electron Spectrosc. Relat. Phenom. 177, 135 (2010).

[27] C. Kolczewski, R. Püttner, O. Plashkevych, H. Ågren, V. Staemmler, M. Martins, G. Snell, A. S. Schlachter, M. Sant'Anna, G. Kaindl, and L. G. M. Pettersson, J. Chem. Phys. 115, 6426 (2001).

[28] C. Kolczewski and K. Hermann, J. Chem. Phys. 118, 7599 (2003).

[29] W. Kutzelnigg, U. Fleischer, and M. Schindler, NMR Basic Principles and Progress (Springer, Berlin, 1990).

[30] M. Nyberg, Ph.D. thesis, Stockholm University, 2000.

[31] L. G. M. Pettersson, U. Wahlgren, and O. Gropen, J. Chem. Phys. 86, 2176 (1987). 
[32] M. Cavalleri, K. Hermann, A. Knop-Gericke, M. Hävecker, R. Herbert, C. Hess, A. Oestereich, J. Döbler, and R. Schlögl, J. Catal. 262, 215 (2009).

[33] T. Sirtl, S. Schlögl, A. Rastgoo-Lahrood, J. Jelic, S. Neogi, M. Schmittel, W. M. Heckl, K. Reuter, and M. J. Lackinger, J. Am. Chem. Soc. 135, 691 (2013).

[34] G. Pawin, K. Wong, D. Kim, D. Sun, L. Bartels, S. Hong, T. Rahman, R. Carp, and M. Marsella, Angew. Chem., Int. Ed. 47, 8442 (2008).

[35] J. Barth, J. Weckesser, N. Lin, A. Dmitriev, and K. Kern, Appl. Phys. A 76, 645 (2003).

[36] C. Perry, S. Haq, B. Frederick, and N. Richardson, Surf. Sci. 409, 512 (1998).

[37] N. Henningsen, R. Rurali, C. Limbach, R. Drost, J. I. Pascual, and K. J. Franke, J. Phys. Chem. Lett. 2, 55 (2011).
[38] Y. Jiang, Q. Huan, L. Fabris, G. C. Bazan, and W. Ho, Nat. Chem. 5, 36 (2012).

[39] F. Albrecht, M. Neu, C. Quest, I. Swart, and J. Repp, J. Am. Chem. Soc. 135, 9200 (2013).

[40] Note that at all bias voltages the same T4PT molecules are distinguished in the $d I / d V$ maps, in contrast to the case of Fe-T4PT networks on $\mathrm{Au}(111)$, where a second monolayer of T4PT molecules sitting atop the Fe atoms has been found [12].

[41] J. Repp, G. Meyer, K.-H. Rieder, and P. Hyldgaard, Phys. Rev. Lett. 91, 206102 (2003).

[42] W. Kuch, M. Salvietti, X. Gao, M.-T. Lin, M. Klaua, J. Barthel, C. V. Mohan, and J. Kirschner, Phys. Rev. B 58, 8556 (1998).

[43] H. Ebert, J. Stöhr, S. S. P. Parkin, M. Samant, and A. Nilsson, Phys. Rev. B 53, 16067 (1996). 